( О.С. Деревянко ${ }^{1 *}$, Л.И. Ибрагимова², М.Р. Рагимов², Т.В. Никонова²

'ООО Лечебный центр, Москва

${ }^{2}$ Научный медицинский исследовательский центр эндокринологии, Москва

В последние годы отмечается рост числа больных с аутоиммунными заболеваниямию Особое внимание уделяется сочетанию нескольких аутоиммунных патологий у одного больного, так как такие пациенты требуют особой тактики ведения. Заболевания желудочно-кишечного тракта аутоиммунной природы являются наименее изученными и, тем не менее, прогностически неблагоприятными. Особое место среди поражений желудка занимает аутоиммунный гастрит (АИГ), развивающийся у больных сахарным диабетом 1 типа (СД1). АИГ - хроническое воспаление слизистой оболочки тела желудка, приводящее к возникновению атрофии и гипосекреции. АИГ в общей популяции встречается, по данным разных авторов, у 5-10\% лиц, при этом установлена более высокая частота встречаемости АИГ у пациентов с СД1 и другой установленной аутоиммунной патологией.

АИГ на ранней стадии заболевания протекает бессимптомно, однако по мере развития атрофических изменений в слизистой оболочке тела желудка у пациентов появляются клинические проявления. Так как течение АИГ не имеет патогномоничных признаков, может проявляться на поздних стадиях гематологическими и онкологическими осложнениями, в связи с чем необходимо проводить скрининг, раннюю диагностику, профилактику и лечение. Наиболее важную роль играют своевременная диагностика и профилактика АИГ и его осложнений.

В данной статье представлен обзор мировых данных, посвященных этой проблеме.

КЛЮЧЕВЫЕ СЛОВА: сахарный диабет 1 типа; аутоиммунный гастрит; аутоиммунные заболевания

\title{
AUTOIMMUNE GASTRITIS: COMORBID PATHOLOGY IN TYPE 1 DIABETES
}

○ Olga S. Derevyanko ${ }^{1 *}$, Liudmila I. Ibragimova², Magomedkerim R. Ragimov², Tatiana V. Nikonova²

'Lechebniy tsentr Ltd, Moscow, Russia

${ }^{2}$ Endocrinology Research Centre, Moscow, Russia

In recent years noted an increased number of patients with autoimmune diseases (AID), and the special attention deserves combination of several autoimmune pathologies collected in one patient, because such patients requires special tactics of management. Autoimmune diseases of the gastrointestinal tract are less researched and, nevertheless, prognostically unfavorable. Autoimmune gastritis (AIG) has a special place in other stomach diseases, which develops in the aggregate with type 1 diabetes. AIG is a chronic inflammation of the mucous membrane of the body of the stomach, leading to the appearance of atrophy and hypoxecretion. AIG is found in $5-10 \%$ of individuals in general population, with a higher incidence of AIG in patients with diabetes and other established autoimmune pathology.

AIG is asymptomatic at the early stage of the disease and clinical manifestations appear after the atrophic changes in the mucous membrane of the stomach develop. Since autoimmune gastritis does not have pathognomonic signs, it can manifest itself in hematological and oncological complications at later stages. This is why screening, early diagnosis, prevention and treatment are very important. Early diagnosis and prevention of AIG and its complications play the main role. This article provides an overview of the worldwide data dedicated to this issue.

KEYWORDS: type 1 diabetes; autoimmune gastritis; autoimmune diseases

В последние годы отмечается рост числа больных с аутоиммунной патологией. Особое внимание уделяется сочетанию нескольких аутоиммунных заболеваний и (АИЗ) у одного больного, так как такие пациенты требуют особой тактики ведения [1].

У пациентов с сахарным диабетом 1 типа (СД1) и у их родственников встречаемость сопутствующих АИЗ значимо выше по сравнению с общей популяцией и составляет, по данным разных авторов, от 10 до 43\% [2].

Органспецифический аутоиммунитет служит основой развития аутоиммунной патологии, что, скорее всего, связано с множественными иммунологическими аномалиями, которые включают в себя определенный дисбаланс в содержании В- и Т-лимфоцитов, либо тенденцию к экспрессивной реакции, направленную против определенных антигенов, либо генетически-опосредованную низкую толерантность к аутоантигенам. Процесс созревания Т-клеток у генетически восприимчивых субъектов происходит путем сложного молекулярного взаимодействия между Т-клеточными рецепторами и HLA-комплексом. «Отбор» рецепторов Т-клеток происходит, вероятно, путем аутоактивации в контексте определенных генов 
HLA-DQ аллелей, которые и являются исходной точкой развития СД1 и других АИЗ. Исходя из всего перечисленного, очевидно, что причины развития аутоиммунной патологии различных органов и систем взаимосвязаны [3].

По сей день роль многих антител к неэндокринным органам еще до конца не изучена, хотя и установлена возможность выявления аутоиммунной патологии задолго до появления клинической симптоматики [4].

Заболевания желудочно-кишечного тракта аутоиммунной природы являются наименее изученными и, тем не менее, прогностически неблагоприятными.

Особое место среди поражений желудка занимает аутоиммунный гастрит (АИГ), развивающийся у больных СД1.

АИГ - хроническое воспаление слизистой оболочки тела желудка с аутоиммунным механизмом развития, приводящее к возникновению атрофического гастрита и гипосекреции [5].

АИГ в общей популяции встречается, по данным разных авторов, у 5-10\% лиц. Однако более высокая частота встречаемости АИГ у пациентов с СД1 и другой установленной аутоиммунной патологией не вызывает сомнений и отмечается во всех работах, посвященных АИГ.

\section{ДИАГНОСТИКА АУТОИММУННОГО ГАСТРИТА}

При АИГ аутоантитела (АТ) к париетальным клеткам желудка (ПКЖ) и продукт их секреции - внутренний фактор Кастла определяются в плазме и желудочном соке [6]. АТ к ПКЖ, циркулирующие в крови больных АИГ, направлены против Н/К-аденозинтрифосфатазы, и их хроническое воздействие на желудок приводит к развитию гипо- или ахлоргидрии, гипергастринемии, а также к железо- и $\mathrm{B}_{12}$-дефицитной анемии.

Кроме того, для оценки состояния слизистой оболочки желудка применяют такие серологические маркеры, как пепсиногены I и II, гастрин-17 [7, 8].

К морфологическим признакам АИГ на ранних этапах заболевания относят лимфоплазмоцитарную инфильтрацию слизистой оболочки желудка, иногда сопровождаемую формированием очаговых лимфоидных скоплений,

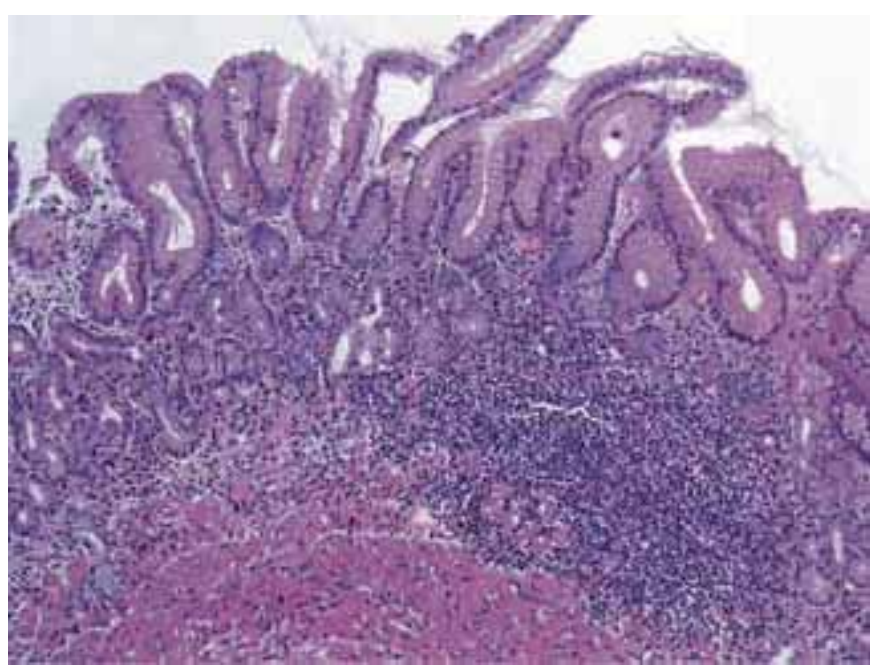

Рис. 1. Аутоиммунный гастрит. Окраска гематоксилином-эозином [5]. а также псевдогипертрофию париетальных клеток. С течением времени развиваются атрофические изменения: уменьшается число желез тела желудка, появляются участки кишечной метаплазии (рис. 1) [9, 10].

\section{ЭТИОЛОГИЯ АУТОИММУННОГО ГАСТРИТА}

Этиология АИГ неизвестна. Очевидна роль генетической предрасположенности, что подтверждается наличием семейных форм АИГ и выявлением в крови АТ к ПКЖ и АИГ у 20-30\% родственников больных пернициозной анемией.

Вероятна связь АИГ с определенными гаплотипами HLA (HLA-B8 и HLA-DR3), однако подобная связь для пернициозной анемии не выявлена. Существуют единичные сообщения о связи пернициозной анемии с гаплотипами HLA-DR4, -DR2 и -DR5 [11]. Часто АИГ встречается в рамках аутоиммунного полигландулярного синдрома (АПС) 2 и 3 типа, сопровождая СД1, АИЗ щитовидной железы, надпочечниковую недостаточность и другие аутоиммунные поражения неэндокринных органов. Предрасположенность к развитию АИГ в рамках АПС определяется в первую очередь наличием гаплотипа DQA 1*0501 [12-14].

Определенная роль пускового фактора, по мнению некоторых авторов, отводится инфекционным агентам - Helicobacter pylori [15], вирусу Эпштейна-Барр (ВЭБ). В частности, было показано, что при хронической инфекции ВЭБ АИГ встречается достоверно чаще (в 48,6\%), чем без нее $(12,1 \%)$, в активную фазу инфекции АТ к ПКЖ выявляются у 64,5\%, а при обнаружении ДНК ВЭБ в биоптатах слизистой оболочки желудка АИГ имеется у 88,6\% [16]. Имеются данные о сочетании H. pylori и инфекции ВЭБ, ухудшающей эрадикацию H. pylori, что приводит к повышению титра АТ к ПЖК и является фактором прогрессирования АИГ [17].

\section{КЛИНИЧЕСКАЯ КАРТИНА}

У большинства пациентов АИГ на ранней стадии заболевания протекает бессимптомно. По мере развития атрофических изменений в слизистой оболочке тела желудка и снижения концентрации витамина $\mathrm{B}_{12}$ у па-

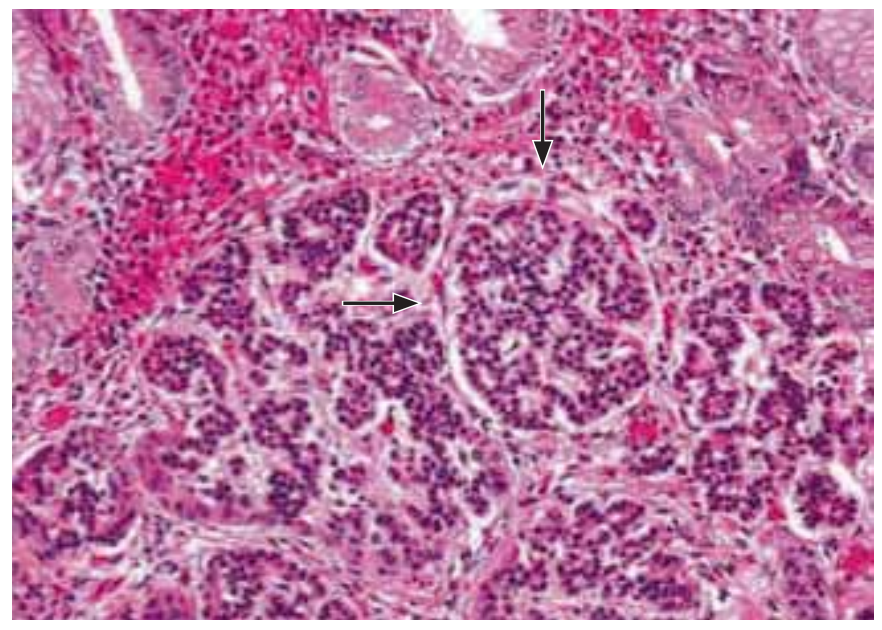

Рис. 2. Карциноид желудка: скопление нейроэндокринных клеток в собственной пластинке слизистой оболочки на фоне аутоиммунного атрофического гастрита с участками кишечной метаплазии. Окраска гематоксилином-эозином [5]. 
циентов появляются клинические признаки пернициозной анемии (гиперхромная макроцитарная анемия) и неврологическая симптоматика (атаксия, слабость в конечностях, парестезии, снижение чувствительности, когнитивные нарушения, деменция). В большинстве случаев диагноз устанавливают только на стадии клинических проявлений, при наличии у пациентов $\mathrm{B}_{12}$-дефицитной анемии, развитие которой свидетельствует о длительном существовании атрофии слизистой оболочки желудка. Возникновение $\mathrm{B}_{12}$-дефицитной анемии у пожилых пациентов может приводить к декомпенсации сердечно-сосудистых заболеваний. Установлено, что неврологические проявления дефицита витамина $\mathrm{B}_{12}$ значимо снижают качество жизни таких пациентов. Значительное повышение содержания гастрина на фоне атрофии слизистой оболочки тела желудка ведет к избыточной стимуляции роста энтерохромаффиноподобных клеток желудка и развитию карциноида желудка, который в 70-80\% случаев наблюдают именно у пациентов с АИГ (рис. 2). Течение карциноида в большинстве случаев бессимптомное, редко приводит к метастазированию [5].

Установлено, что у пациентов с АИГ риск развития рака желудка в 2-4 раза выше, чем в общей популяции [5].

\section{АУТОИММУННЫЙ ГАСТРИТ И САХАРНЫЙ ДИАБЕТ 1 ТИПА}

В проведенном нами исследовании у пациентов с СД1 без клинических проявлений гастрита АТ к ПКЖ были выявлены у 43\% обследованных, при этом важно отметить, что в группе контроля (лиц без установленных АИЗ) данные антитела не встречались вообще [2].

Установлено, что риск развития АИГ повышается с возрастом и длительностью СД1, пик заболеваемости приходится на возраст 60 лет и старше, женщины заболевают в 4-5 раз чаще мужчин $[3,4,11]$. Однако имеются данные о встречаемости АИГ у детей. В исследовании А.А. Звягина отмечено, что у 15,4\% детей с СД1 были выявлены АТ к ПКЖ, характерные для АИГ [18].

При обследовании 108 детей с СД1 (61 девоч-

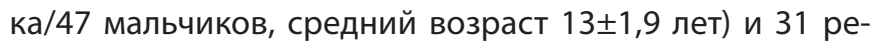
бенка с хроническим гастритом без СД1, АТ к ПКЖ были обнаружены у 10,1\% детей с СД1. Продолжительность СД1 во всех случаях составила более 10 лет. У одного из пациентов с наличием АТ к ПКЖ была обнаружена инфекция Helicobacter pylori. При этом гипергастринемия была диагностирована у всех пациентов с наличием АТ к ПКЖ [19].

В 2016 г. в журнале ВMJ опубликована статья, в которой ученые Медицинского университета им. Нельсона Манделы из ЮАР проводили исследование, целью которого являлось выявление у больных аутоиммунного тиреоидита, АИГ, пернициозной анемии на фоне СД1.

В исследуемую группу входили 202 пациента, принадлежащие к различным этническим группам: африканской расы (56,4\%; $n=114)$, индийцы $(31,7 \% ; n=64)$, европеоидной расы $(4,5 \% ; n=9)$ и метисы $(7,4 \% ; n=15)$. Возраст и длительность заболевания СД1 варьирова-

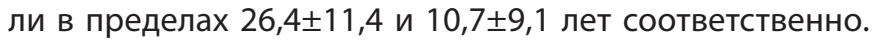

Всем больным были определены АТ к ПКЖ. По результатам исследования АТ были выявлены у 8,9\% (n=17). Дефицит витамина $\mathrm{B}_{12}$ был диагностирован у $3,5 \%(\mathrm{n}=7)$, железодефицитная анемия у $19,3 \%(n=39)$ соответственно [20].

Еще одно проспективное исследование проводили специалисты из Департамента клинической и экспериментальной медицины университета Пизы в 2016 г. В группу исследования вошли 3069 пациентов, разделенных на 2 группы - без нарушения функции щитовидной железы и с ранее диагностированным диффузным токсическим зобом. Примечательно, что для больных с болезнью Грейвса было характерно развитие и других аутоиммунных патологий, таких как АИГ (2,8\%), болезнь Аддисона (0,9\%), витилиго (2,7\%), ревматоидный артрит $(2,4 \%)$, целиакия $(1,4 \%)$ и др. Наиболее часто встречались следующие комбинации АИЗ: аутоиммунный тиреоидит + АИГ + витилиго + СД1 (2,2\%) и аутоиммунный тиреоидит + АИГ + ревматоидный артрит + СД1 $(1,9 \%)[21]$.

В 2016 г. в журнале Cureus была опубликована статья сотрудников национального университета Перу (Carlos Culquichicón-Sánchez, Ricardo Correa, Igor Flores-Guevara, Frank Espinoza Morales, Christian R Mejia), которые описали редкий клинический случай развития тромбоцитопенической пурпуры на фоне СД1 и хеликобактер-ассоциированного гастрита у мальчика 11 лет. Авторы указывают на то, что подобный случай лишь третий выявленный в Латинской Америке и пятнадцатый в мире. Авторы также не исключают возможность связи развития АИГ и, как следствие, пернициозной анемии с СД1 и тромбоцитопенической пурпурой [22].

В 2017 г. в Скандинавском журнале гастроэнтерологии было опубликовано исследование сотрудников итальянского департамента молекулярной медицины г. Перуджа, целью которого являлось проведение параллели между АИГ и другими АИЗ. В исследовании участвовали 138 пациентов (48 мужчин, чей возраст варьировал от 27 до 83 лет, и 90 женщин в возрасте от 15 лет до 91 года). У 11 пациентов в ходе данного исследования был впервые выявлен СД1 (7,8\% - 4 мужчины и 7 женщин), у 32 (23\% - 25 женщин, 7 мужчин) выявлен аутоиммунный тиреоидит, у 3 - витилиго (1 женщина, 2 мужчин - 2,8\%). АИГ выявлены у 90 больных (65\% - 64 женщины, 26 мужчин) и пернициозная анемия у 35 (25\% - 21 женщина, 14 мужчин) пациентов соответственно. Примечателен тот факт, что у 4 пациентов с диагностированным в ходе исследования СД1 были обнаружены АТ к ПКЖ [23]. По данным Бельгийского регистра диабета [24], проводилось исследование с целью выявления распространенности АТ к ПКЖ у детей и взрослых с ранее диагностированным СД1. В результате были обнаружены АТ к ПКЖ у 10-15\% детей и 15-25\% взрослых, при этом комплекс АИГ + пернициозная анемия был выявлен у 5-10 и 2,5-4\% больных СД1 по сравнению с 2 и 0,5-1\% соответственно в общей популяции.

\section{ЗАКЛЮЧЕНИЕ}

Анализируя все вышеперечисленные работы, можно сделать вывод, что проблема АИЗ, протекающих на фоне 
СД1, остается актуальной в настоящее время. АИГ относится к наименее изученным, но вместе с тем прогностически неблагоприятным АИЗ.

В настоящее время этиологического и патогенетического лечения АИГ не существует. Большое значение придают своевременной диагностике дефицита витамина $\mathrm{B}_{12}$ и профилактике $\mathrm{B}_{12}$-дефицитной анемии. Кроме того, пациентам с АИГ показано регулярное выполнение эндоскопического исследования верхних отделов жКТ с биопсией слизистой оболочки желудка с целью ранней диагностики рака желудка [25]. Учитывая, что течение АИГ не имеет патогномоничных признаков, и он проявляется на поздних стадиях развитием гематологических и онкологических осложнений, необходимо проводить скрининг, раннюю диагностику, профилактику и лечение.

\section{ДОПОЛНИТЕЛЬНАЯ ИНФОРМАЦИЯ}

Источник финансирования. Поисково-аналитическая работа по подготовке рукописи проведена при финансовой поддержке Российского научного фонда (проект №17-75-30035).

Конфликт интересов. Авторы декларируют отсутствие явных и потенциальных конфликтов интересов, связанных с публикацией настоящей статьи.

Участие авторов. О.С. Деревянко - обоснование рукописи, поиск данных по заданной теме, проверка критически важного интеллектуального содержания; Л.И. Ибрагимова - поиск данных по заданной теме, проверка критически важного интеллектуального содержания; М.Р. Рагимов - поиск данных по заданной теме; Т.В. Никонова - окончательное утверждение для публикации рукописи. Все авторы внесли существенный вклад в проведение исследования и подготовку статьи, прочли и одобрили финальную версию перед публикацией.

\section{СПИСОК ЛИТЕРАТУРЫ | REFERENCES}

1. Anaya JM. The autoimmune tautology. A summary of evidence. Joint Bone Spine. 2017;84(3):251-253. doi: 10.1016/j.jbspin.2016.11.012

2. Деревянко О.С. Аутоантитела к неэндокринным органам при сахарном диабете 1 mипа: диагностика, оченка клинической значимости и прогноза: Автореф. дис. ... канд. мед. наук. - М.; 2014. [Derevyanko OS. Autoantitela kneendokrinnym organam pri sakharnom diabete 1 tipa: diagnostika, otsenka klinicheskoy znachimosti i prognoza. [dissertation] Moscow; 2014. (In Russ.)]

3. Kahaly GJ, Hansen MP. Type 1 diabetes associated autoimmunity. Autoimmun Rev. 2016;15(7):644-648. doi: 10.1016/j.autrev.2016.02.017

4. Toh BH. Diagnosis and classification of autoimmune gastritis. Autoimmun Rev. 2014;13(4-5):459-462. doi: 10.1016/j.autrev.2014.01.048

5. Ивашкин В.Т., Шептулина А.Ф., Райхельсон КЛ., и др. Аутоимунные заболевания органов пищеварительной системы // Вестник Российской академии медичинских наук. - 2015. T. 70. - №2. - C. 139-151. [Ivashkin VT, Sheptulina AF, Raikhel'son $\mathrm{KL}$, et al. Autoimmune Diseases of Digestive System. Annals of the Russian Academy of Medical Sciences. 2015;70(2):139-151. (In Russ.)] doi: 10.15690/vramn.v70.i2.1306

6. Barrett SP, Riordon A, Toh BH, et al. Homing and adhesion molecules in autoimmune gastritis. J Leukoc Biol. 2000;67(2):169-173. doi: 10.1002/jlb.67.2.169

7. Varbanova M, Frauenschlager K, Malfertheiner P. Chronic gastritis an update. Best Pract Res Clin Gastroenterol. 2014;28(6):1031-1042. doi: 10.1016/j.bpg.2014.10.005

8. Whittingham S, Mackay IR. Autoimmune gastritis: historical antecedents, outstanding discoveries, and unresolved problems. Int Rev Immunol. 2005;24(1-2):1-29. doi: 10.1080/08830180590884413

9. Lee JY, Kim N, Lee HS, et al. Correlations among endoscopic, histologic and serologic diagnoses for the assessment of atrophic gastritis. J Cancer Prev. 2014;19(1):47-55.

10. Polydorides AD. Pathology and differential diagnosis of chronic, noninfectious gastritis. Semin Diagn Pathol. 2014;31 (2):114-123. doi: 10.1053/j.semdp.2014.02.008

11. De Block CE, De Leeuw IH, Van Gaal LF. Autoimmune gastritis in type 1 diabetes: a clinically oriented review. J Clin Endocrinol Metab. 2008;93(2):363-371. doi: 10.1210/jc.2007-2134

12. Репина Е.А. Общие генетические маркеры сахарного диабета 1-го типа и аутоиммунных заболеваний щитовидной железы // Сахарный диабет. - 2011. - T. 14. - №2. - C. 23-31. [Repina EA. The role of common genetic markers in susceptibility to type 1 diabetes and autoimmune thyroid diseases. Diabetes Mellitus. 2011;14(2):23-31. (In Russ.)] doi: 10.14341/2072-0351-5630

13. Golden B, Levin $L$, Ban $Y$, et al. Genetic analysis of families with autoimmune diabetes and thyroiditis: evidence for common and unique genes. J Clin Endocrinol Metab. 2005;90(8):4904-4911. doi: $10.1210 /$ jc.2004-2236

14. Villano MJ, Huber AK, Greenberg DA, et al. Autoimmune thyroiditis and diabetes: dissecting the joint genetic susceptibility in a large cohort of multiplex families. $J$ Clin Endocrinol Metab. 2009;94(4):1458-1466. doi: 10.1210/jc.2008-2193

15. Smyk DS, Koutsoumpas AL, Mytilinaiou MG, et al. Helicobacter pylor and autoimmune disease: cause or bystander. World J Gastroenterol. 2014;20(3):613-629. doi: 10.3748/wjg.v20.i3.613

16. Волынец Г.В. Клинические и диагностические особенности и принципы терапии аутоиммунного гастрита у детей // Детская гастроэнтерология. - 2005. - №3. - С. 33-37. [Volynets GV. Klinicheskie i diagnosticheskie osobennosti i printsipy terapii autoimmunnogo gastrita u detey. Detskaya gastroenterologiya. 2005;(3):33-37. (In Russ.)]

17. Новикова В.П. Этиопатогенетические и клинико-морфологические особенности хронического гастрита в разном возрасте: Автореф. дис. ... д-ра мед. наук. - СПб.; 2009. [Novikova VP. Etiopatogeneticheskie i kliniko-morfologicheskie osobennosti khronicheskogo gastrita v raznom vozraste. [dissertation] Saint-Petersburg; 2009. (In Russ.)]

18. Звягин А.А. Аутоиммунный гастрит и детей с аутоиммунными заболеваниями // Педиатр. - 2013. - Т. 4. - №4. - С. 44-47. [Zvyagin AA. Autoimmune gastritis in children with autoimmune diseases. Pediatrician (St. Petersburg). 2013;4(4):44-47. (In Russ.)]

19. Нижевич АА. Якупова Г.М., Малиевский О.А., и др. Аутоиммунный гастрит у детей с сахарным диабетом 1 типа // Экспериментальная и клиническая гастроэнтерология. - 2016. - №1. - С. 28-33. [Nizhevitch AA, Yakupova GM, Malievsky OA, et al. Autoimmune gastritis in children with type 1 diabetes. Experimental and clinical gastroenterology. 2016;(1):28-33. (In Russ.)]

20. Paruk IM, Ganie Y, Maharaj S, et al. High prevalence of antithyroid peroxidase and antiparietal cell antibodies among patients with type 1 diabetes mellitus attending a tertiary diabetes centre in South Africa. Postgrad Med J. 2017;93(1100):338-343. doi: 10.1136/postgradmedj-2016-134420

21. Fallahi P, Ferrari SM, Ruffilli I, et al. The association of other autoimmune diseases in patients with autoimmune thyroiditis: Review of the literature and report of a large series of patients. Autoimmun Rev. 2016;15(12):1125-1128. doi: 10.1016/j.autrev.2016.09.009

22. Culquichicon-Sanchez C, Correa R, Flores-Guevara I, et al. Immune Thrombocytopenic Purpura and Gastritis by H. pylori Associated With Type 1 Diabetes Mellitus. Cureus. 2016;8(2):e512. doi: 10.7759/cureus.512

23. Villanacci V, Casella G, Lanzarotto F, et al. Autoimmune gastritis: relationships with anemia and Helicobacter pylori status. Scand J Gastroenterol. 2017;52(6-7):674-677. doi: 10.1080/00365521.2017.1288758

24. De Block CEM, De Leeuw IH, Van Gaal LF. High Prevalence of Manifestations of Gastric Autoimmunity in Parietal Cell Antibody- Positive Type 1 (Insulin-Dependent) Diabetic Patients 1. J Clin Endocrinol Metab. 1999;84(11):4062-4067. doi: 10.1210/jcem.84.11.6095

25. Vannella L, Lahner E, Annibale B. Risk for gastric neoplasias in patients with chronic atrophic gastritis: a critical reappraisal. World J Gastroenterol. 2012;18(12):1279-1285. doi: 10.3748/wjg.v18.112.1279 


\section{ИНФОРМАЦИЯ ОБ АВТОРАХ [AUTHORS INFO]}

*Деревянко Ольга Сергеевна, к.M.н. [Olga S. Derevyanko, MD, PhD]; адрес: Россия, 119021, Москва, ул. Тимура Фрунзе, д. 15/1 [15/1 Timura Frunze st, Moscow, 119021 Russian Federation] ORCID: http://orcid.org/0000-0002-3222-6990; eLibrary SPIN: 9977-7856; e-mail: olgader14@mail.ru

Ибрагимова Людмила Ибрагимовна, K.M.H. [Liudmila I. Ibragimova, MD, PhD]; ORCID: http://orcid.org/0000-0003-3535-520X; eLibrary SPIN: 5013-8222; e-mail: ibragimovaliudmila@gmail.com Рагимов Магомедкерим Разинович, аспирант [Magomedkerim R. Ragimov, MD, PhD student]; ORCID: http://orcid.org/0000-0002-4290-3182; eLibrary SPIN: 8445-6795; e-mail: tga.endocrine@gmail.com Никонова Татьяна Васильевна, д.м.н. [Tatiana V. Nikonova, MD, PhD]; ORCID: http://orcid.org/0000-0001-5656-2596; eLibrary SPIN: 8863-0201; e-mail: tatiana_nikonova@mail.ru

\section{ЦИТИРОВАТЬ:}

Деревянко О.С., Ибрагимова Л.И., Рагимов М.Р., Никонова Т.В. Аутоиммунный гастрит как коморбидная патология при сахарном диабете 1 типа // Сахарный диабет. — 2018. - T. 21. — №5. - C. 404-408. doi: 10.14341/DM9465

\section{TO CITE THIS ARTICLE:}

Derevyanko OS, Ibragimova LI, Ragimov MP, Nikonova TV. Autoimmune gastritis: comorbid pathology in type 1 diabetes. Diabetes Mellitus. 2018;21(5):404-408. doi: 10.14341/DM9465 